\title{
Simple moving-average formulae for the direct recovery of the relaxation spectrum
}

\author{
R. S. Anderssen \\ CSIRO Mathematical and Information Sciences, GPO Box 664, Canberra, \\ ACT 2601, Australia
}

A. R. Davies ${ }^{\text {a) }}$

Institute of Non-Newtonian Fluid Mechanics, Department of Mathematics, University of Wales, Aberystwyth, SY23 3BZ, United Kingdom

(Received 1 March 2000; final revision received 16 October 2000)

\section{Synopsis}

Different software packages are available commercially which can be applied to oscillatory shear data to recover an estimate of the relaxation spectrum of the viscoelastic material tested. The underlying algorithms, based on some form of regularization, are indirect and technically involved. Davies and Anderssen [J. Non-Newtonian Fluid Mech. 73, 163-179 (1997)] have derived exact sampling localization results for the determination of elastic moduli from (exact) storage and loss moduli. It is now shown how their results can be exploited to construct simple and explicit moving-average formulae which recover estimates of the relaxation spectrum from oscillatory shear data, with realistic observational errors. Explicit moving-average formulae are presented which experimentalists can apply immediately to appropriately sampled oscillatory shear measurements. The given formulae are validated on noisy data obtained from synthetic relaxation spectra. (c) 2001 The Society of Rheology. [DOI: 10.1122/1.1332787]

\section{INTRODUCTION}

Many methods have been proposed for estimating the discrete relaxation spectrum $\left\{\tau_{j}, g_{j}\right\}$ of a viscoelastic fluid from oscillatory shear measurements. The majority of these methods attempt, in various ways, to fit the dual model

$$
\begin{aligned}
& G_{N}^{\prime}(\omega)=\sum_{j=1}^{N} g_{j} \frac{\omega^{2} \tau_{j}^{2}}{1+\omega^{2} \tau_{j}^{2}}, \\
& G_{N}^{\prime \prime}(\omega)=\sum_{j=1}^{N} g_{j} \frac{\omega \tau_{j}}{1+\omega^{2} \tau_{j}^{2}},
\end{aligned}
$$

to measurements of the storage and loss moduli, $G^{\prime}(\omega)$ and $G^{\prime \prime}(\omega)$, respectively. In (1), $\tau_{j}$ denotes the relaxation time, and $g_{j}$ the elastic modulus, associated with the $j$ th Maxwell mode. The corresponding approximations to the linear relaxation modulus, $G(t)$, and the continuous relaxation spectrum, $H(\tau)$, take the form

\footnotetext{
a) Author to whom correspondence should be addressed. Electronic mail: ard@aber.ac.uk
} 


$$
G_{N}(t)=\sum_{j=1}^{N} g_{j} \exp \left(-t / \tau_{j}\right),
$$

and

$$
H(\tau)=\sum_{j=1}^{N} \eta_{j} \delta\left(\tau-\tau_{j}\right),
$$

where $\eta_{j}=g_{j} \tau_{j}$ denotes the partial viscosity associated with the $j$ th Maxwell mode, and $\delta\left(\tau-\tau_{j}\right)$ is the delta-function centered at $\tau=\tau_{j}$.

The problem of fitting the model (1) and (2) to given measurements of $G^{\prime}(\omega)$ and $G^{\prime \prime}(\omega)$ is ill posed, for the same reason that the problem of inverting the Fredholm integral equations

$$
\begin{aligned}
& G^{\prime}(\omega)=\int_{0}^{\infty} H(\tau) \frac{\omega^{2} \tau^{2}}{1+\omega^{2} \tau^{2}} \frac{d \tau}{\tau}, \\
& G^{\prime \prime}(\omega)=\int_{0}^{\infty} H(\tau) \frac{\omega \tau}{1+\omega^{2} \tau^{2}} \frac{d \tau}{\tau},
\end{aligned}
$$

which are the continuous counterparts of (1) and (2), is also ill posed. The mathematical aspects of this subject are treated in many books including Engl et al. (1996) and Groetsch (1984).

Algorithms for determining the discrete spectrum $\left\{\tau_{j}, g_{j}\right\}$ use various techniques to stabilize the inherent ill posedness. Honerkamp and Weese (1989, 1990, and 1993), and Mead (1994), use different forms of regularization, under the assumption that the relaxation times $\tau_{j}$ are known, and thereby recover a model with a large number $N$ of modes. On the other hand, Baumgaertel and Winter (1989) achieve stabilization through leastsquares fitting the fully nonlinear model (1) and (2) for a small number $N$ of the modes. Yanovsky et al. (1996) advocate the use of fitting in the uniform norm, rather than a Sobolev norm, while Emri and Tschoegl (1993), and Brabec and Schausberger (1995), achieve stabilization through various filtering routines. Yet another approach, based on the Laplace transform, is proposed by Carrot and Verney (1996). Here, stabilization is achieved by controlling the order of a Padé approximant.

The main difficulty with all such methods is that they rely on the choice of a stabilizing functional, one or more regularization parameters, or a filter. The resulting accuracy of the recovered spectrum is controlled by these choices, and, therefore, is not necessarily optimal. For example, the parsimonious approach used by Baumgaertel and Winter (1989) to determine $N$, the number of modes to be fitted to the available data, is an application of the much-used Morozov discrepancy principle [Morozov (1966)], which is at best only weakly optimal [Davies (1992)].

No practical method of determining the spectrum can be free of control parameters. The advantage of the approach introduced in this paper is that of great simplicity of implementation. The aim is to compute mean values of the relaxation spectrum over small intervals of relaxation times $a<\tau<b$ (or, equivalently, $\ln a<\ln \tau<\ln b$ ), where $0<a<b<\infty$. For this purpose, Davies and Anderssen (1997) defined elastic moduli

$$
g_{a b}=\int_{a}^{b} H(\tau) \frac{d \tau}{\tau}=\int_{\ln a}^{\ln b} H(\tau) d(\ln \tau)
$$


for the intervals $(a, b)$ or $(\ln a, \ln b)$ over which the mean values are taken.

Once estimates for the elastic moduli have been obtained, on some grid of relaxation times or the logarithms of relaxation times, they can be interpreted and utilized in various ways to construct approximations to the relaxation spectrum $H(\tau)$.

In this paper, the goal is the construction of moving-average formulas which can be applied directly to given measurements of the storage and loss moduli $G^{\prime}(\omega)$ and $G^{\prime \prime}(\omega)$, in order to estimate the various elastic moduli of Eq. (7). This is achieved by exploiting a theoretical result of Davies and Anderssen (1997) that, provided $\ln (b / a)$

$<\pi$, the elastic modulus $g_{a b}$, defined by (7), has the following data-functional representations:

$$
\begin{gathered}
g_{a b} \equiv g_{a b}^{\prime}=-\int_{-\ln b-\pi / 2}^{-\ln a+\pi / 2}\left\{E^{\prime \prime}[\ln (b \omega)]-E^{\prime \prime}[\ln (a \omega)]\right\} G^{\prime}(\omega) d(\ln \omega), \\
g_{a b} \equiv g_{a b}^{\prime \prime}=\int_{-\ln b-\pi / 2}^{-\ln a+\pi / 2}\left\{E^{\prime}[\ln (b \omega)]-E^{\prime \prime}[\ln (a \omega)]\right\} G^{\prime \prime}(\omega) d(\ln \omega),
\end{gathered}
$$

with

$$
E^{\prime}(x)=\lim _{\epsilon \rightarrow 0} E_{\epsilon}^{\prime}(x), \quad E^{\prime \prime}(x)=\lim _{\epsilon \rightarrow 0} E_{\epsilon}^{\prime \prime}(x)
$$

where the functions $E_{\epsilon}^{\prime}(x)$ and $E_{\epsilon}^{\prime \prime}(x)$ are the real and imaginary components of the elementary sampling function

$$
E_{\epsilon}^{*}(x)=E_{\epsilon}^{\prime}(x)+i E_{\epsilon}^{\prime \prime}(x) \equiv \frac{1}{\pi} \operatorname{erf}\left(\frac{x+\frac{1}{2} \pi i}{\sqrt{2} \epsilon}\right), \quad \epsilon>0 .
$$

In (8), the notation $g_{a b}^{\prime}$ indicates that the elastic modulus $g_{a b}$ is obtained directly from $G^{\prime}(\omega)$, the storage modulus, while, in (9), $g_{a b}^{\prime \prime}$ indicates that $g_{a b}$ is obtained directly from $G^{\prime \prime}(\omega)$, the loss modulus. In theory, if $G^{\prime}(\omega)$ and $G^{\prime \prime}(\omega)$ were known exactly as continuous functions of $\omega$, then $g_{a b}^{\prime}$ and $g_{a b}^{\prime \prime}$ would both generate the same value. In practice, this is not possible, since the storage and loss moduli are only available as inexact, discrete measurements.

Formulae (8) and (9) make explicit use of the sampling localization theorems derived by Davies and Anderssen (1997); namely, provided that $\ln (b / a)<\pi$, the elastic modulus on the interval $(a, b)$ is determined solely from the values of $G^{\prime}(\omega)$ and $G^{\prime \prime}(\omega)$ at the frequencies in the range $\left[\exp \left(-\frac{1}{2} \pi / b\right), \exp \left(\frac{1}{2} \pi / a\right)\right]$ (or, equivalently, in the range $-\ln b$ $-\frac{1}{2} \pi<\ln \omega<-\ln a+\frac{1}{2} \pi$ ). Starting from (8) and (9), it is shown below how to construct and apply moving-average formulae which enable one to recover the relaxation spectrum directly from experimental measurements of the storage and loss moduli.

\section{CONTINUOUS AND DISCRETE MOVING-AVERAGE FORMULAE FOR THE ELASTIC MODULI}

Equations (8) and (9), for the elastic moduli, can be transformed to continuous moving-average formulae using the transformation

$$
x=\ln \omega+\frac{1}{2} \ln (a b), \quad \Delta=\ln \left(\frac{b}{a}\right), \quad 0<\Delta<\pi .
$$


They become, respectively,

$$
\begin{gathered}
g_{a b} \equiv g_{a b}^{\prime}=-\int_{-\frac{1}{2}(\pi+\Delta)}^{\frac{1}{2}(\pi+\Delta)}\left[E^{\prime \prime}\left(x+\frac{1}{2} \Delta\right)-E^{\prime \prime}\left(x-\frac{1}{2} \Delta\right)\right] G^{\prime}\left(\frac{e^{x}}{\sqrt{a b}}\right) d x, \\
g_{a b} \equiv g_{a b}^{\prime \prime}=\int_{-\frac{1}{2}(\pi+\Delta)}^{\frac{1}{2}(\pi+\Delta)}\left[E^{\prime}\left(x+\frac{1}{2} \Delta\right)-E^{\prime}\left(x-\frac{1}{2} \Delta\right)\right] G^{\prime \prime}\left(\frac{e^{x}}{\sqrt{a b}}\right) d x .
\end{gathered}
$$

The discrete moving-average formulae presented in this paper are derived by applying product-integration to the earlier continuous moving-average formulae. Initially, the nature of the discretization to be utilized must be defined. This must reflect the fact that, experimentally, the storage and loss moduli $G^{\prime}$ and $G^{\prime \prime}$ will only be measured at a discrete set of $K+1$ frequencies

$$
\omega_{k}, \quad k=0,1,2, \cdots, K,
$$

which are assumed to increase as a function of $k$. Once the discretization is specified, the form of the product-integration to be applied can be defined.

\section{A. The discretization}

The discrete moving-average formulae, to be constructed from (13) and (14), will take the form

$$
\begin{gathered}
g_{j}^{\prime}=-\sum_{l=-L}^{L} \alpha_{l} G^{\prime}\left(\omega_{j+l}\right), \quad j=L, L+1, \cdots, K-L, \\
g_{j}^{\prime \prime}=\sum_{l=-L}^{L} \beta_{l} G^{\prime \prime}\left(\omega_{j+l}\right), \quad j=L, L+1, \cdots, K-L,
\end{gathered}
$$

where $L$ is an integer not less than 2 , and the $\alpha_{l}$ and $\beta_{l}$ denote constant coefficients. The discrete elastic moduli $g_{j}^{\prime}$ and $g_{j}^{\prime \prime}$ will be associated with the relaxation time $\tau_{j}$ defined by

$$
\tau_{j}=\omega_{j}^{-1}, \quad j=L, L+1, \cdots, K-L .
$$

It is important to recall at this stage that, if the storage and loss moduli are sampled at frequencies in the range

$$
\omega_{0} \leqslant \omega \leqslant \omega_{K},
$$

then the relaxation modes (identified by the subscript " $j$ ") are recoverable only in the reduced reciprocal range

$$
\omega_{K-L}^{-1} \leqslant \tau \leqslant \omega_{L}^{-1}
$$

That it is impossible to recover information about the relaxation spectrum in the full reciprocal range $\omega_{K}^{-1} \leqslant \tau \leqslant \omega_{0}^{-1}$ is a direct consequence of sampling localization [Davies and Anderssen (1997)]. The range of the index $j$ in (16)-(18) is deliberately chosen to reflect the reduced limits. For example, if $K=28$ (29 sampling points) and $L=4$, no more than 21 relaxation modes can be recovered using the moving-average formulae (16) and (17).

The length of the moving-average formulae is defined to be $2 L+1$. Alternatively, these formulae can be referred to as $(2 L+1)$-point formulae, since each mode (identified by $j$ ) will recover information from (at most) $2 L+1$ sampled frequencies. 
For (16) and (17), the length $2 L+1$ is determined by the following two factors:

(1) The length $\Delta=\ln (b / a)$ of the interval of relaxation times $a<\tau<b$ over which the elastic modulus $g_{a b}$ is defined. This interval $(a, b)$ will be called the averaging interval for reasons which will be explained later.

(2) The rate at which the frequencies $\omega_{k}$ are sampled.

In order to establish a correspondence between the continuous formulae (13) and (14) and their discrete counterparts (16) and (17), the geometric center $\sqrt{a b}$ of the averaging interval $(a, b)$ is chosen to coincide with the discrete relaxation time $\tau_{j}$; i.e.,

$$
\tau_{j}=\sqrt{a b}, \quad \text { or } \quad \omega_{j}=\frac{1}{\sqrt{a b}} .
$$

It is also necessary to ensure that there is a local grid $\left\{x_{l}\right\},-L \leqslant l \leqslant L$, at which the arguments of $G^{\prime}$ and $G^{\prime \prime}$ in (13) and (14) and in (16) and (17) are matched; i.e.,

$$
\frac{\exp \left(x_{l}\right)}{\sqrt{a b}}=\omega_{j+l}, \quad \text { or } \quad x_{l}=\ln \left(\frac{\omega_{j+l}}{\omega_{j}}\right) .
$$

This is possible if and only if the measurement frequencies $\omega_{k}$ are exponentially sampled; i.e.,

$$
\omega_{k}=\omega_{0} \exp (k h), \quad 0 \leqslant k \leqslant K,
$$

where the constant $h$ is called the sampling interval. Exponential sampling clearly corresponds to uniform sampling in the log-frequency domain; i.e.,

$$
\ln \left(\omega_{k}\right)=\ln \left(\omega_{0}\right)+k h, \quad h=\ln \left(\frac{\omega_{k+1}}{\omega_{k}}\right) .
$$

The local grid points (20) then take the form

$$
x_{l}=l h, \quad-L \leqslant l \leqslant L,
$$

and coincide locally with the uniformly-spaced log-frequency grid $\left\{\ln \left(\omega_{k}\right)\right\}$. The central point $x_{0}=0$ corresponds to the point $\ln \left(\omega_{j}\right)$ which itself corresponds to the central term $(l=0)$ in both (16) and (17).

Exponential sampling, coupled with the translational invariance of the formulae (13) and (14) with respect to $(\ln a, \ln b)$, ensures that the coefficients $\alpha_{l}$ and $\beta_{l}$ in (16) and (17) are independent of the $j$ th mode or the $j$ th relaxation time. The coefficients depend only on the half-length $L$ of the formulae. In particular, they do not depend on the oscillatory shear data.

The way in which the coefficients $\alpha_{l}$ and $\beta_{l}$ are determined is explained in subsequent subsections. A rigorous derivation would be quite technical, so only formal arguments are presented. Once these coefficients are determined, however, the numerical implementation of the resulting moving-averages takes a matter of seconds of programming time. Estimates of the relaxation spectrum are then computed in a fraction of a second.

Notation. In the discrete setting, the interval of integration in (8) and (9) will be centered at the $\log$-frequency points $\ln \omega_{j}$, while the quantities $g_{a b}^{\prime}$ and $g_{a b}^{\prime \prime}$ are replaced by their discrete approximations $g_{j}^{\prime}$ and $g_{j}^{\prime \prime}$, respectively. 


\section{B. Product midpoint integration}

Without loss of generality, the product-integration process will only be outlined for the calculation of the elastic moduli $g_{j}^{\prime}$ from the storage modulus $G^{\prime}(\omega)$. A similar argument to that constructed later applies to the calculation of the elastic moduli $g_{j}^{\prime \prime}$ from the loss modulus $G^{\prime \prime}(\omega)$. The experimentally sampled storage moduli $G^{\prime}\left(\omega_{k}\right)$ are used to construct the following approximation to $G^{\prime}(\omega)$ :

$$
\hat{G}^{\prime}(\omega)=\sum_{k=0}^{K} G^{\prime}\left(\omega_{k}\right) \phi_{k}(\omega),
$$

where the $\phi_{k}(\omega)$ represent the box functions

$$
\phi_{k}(\omega)=\left\{\begin{array}{ll}
1, & \text { if } \exp (-h / 2) \omega_{k}<\omega<\exp (h / 2) \omega_{k} \\
0, & \text { otherwise }
\end{array} .\right.
$$

Substituting (24) into (8), before taking the limit as $\epsilon \rightarrow 0$ in (10), one obtains, after applying the change of variable $x=\ln \left(\omega / \omega_{j}\right)$,

$$
g_{j, \Delta}^{\prime}(\epsilon)=-\sum_{l-L}^{L} w_{l}^{\prime \prime}(\epsilon) G^{\prime}\left(\omega_{j+l}\right),
$$

with

$$
w_{l}^{\prime \prime}(\epsilon)=\int_{(l-1 / 2) h}^{(l+1 / 2) h}\left[E_{\epsilon}^{\prime \prime}\left(x+\frac{1}{2} \Delta\right)-E_{\epsilon}^{\prime \prime}\left(x-\frac{1}{2} \Delta\right)\right] d x .
$$

For finite $\epsilon>0$, equations (26) and (27) provide the product midpoint integration rule for (8), with the geometric center $\sqrt{a b}$ coincident with $\omega_{j}^{-1}$. The relationship between the half-length $L$ of the formula (26) and the sampling interval $h$ will be discussed later.

The counterpart for (9) is given by

$$
g_{k, \Delta}^{\prime \prime}(\epsilon)=\sum_{l=-L}^{L} w_{l}^{\prime}(\epsilon) G^{\prime \prime}\left(\omega_{k+l}\right),
$$

where

$$
w_{l}^{\prime}(\epsilon)=\int_{(l-1 / 2) h}^{(l+1 / 2) h}\left[E_{\epsilon}^{\prime}\left(x+\frac{1}{2} \Delta\right)-E_{\epsilon}^{\prime}\left(x-\frac{1}{2} \Delta\right)\right] d x .
$$

\section{Choosing the sampling and averaging intervals}

It follows from the original definition (7) that, when $H(\tau)$ is continuous, the elastic modulus $g_{a b}$ can be given the following mean-value interpretation, with respect to the $(\ln \tau)$-measure

$$
g_{a b}=\int_{\ln a}^{\ln b} H(\tau) d(\ln \tau)=H\left(\tau^{*}\right) \ln (b / a),
$$

where $\tau^{*} \in(a, b)$ denotes any one of the relaxation times where the mean-value $H\left(\tau^{*}\right)$ is achieved. Since, for $g_{j}^{\prime}$ and $g_{j}^{\prime \prime}$, 


$$
\tau_{j}=\omega_{j}^{-1}=\sqrt{a b}, \quad \text { and } \quad \Delta=\ln \left(\begin{array}{c}
b \\
\frac{a}{a}
\end{array}\right),
$$

the counterparts of the earlier mean-value interpretation become

$$
H\left(\bar{\tau}_{j}^{\prime}\right)=g_{j, \Delta}^{\prime} / \Delta, \quad \text { and } \quad H\left(\bar{\tau}_{j}^{\prime \prime}\right)=g_{j, \Delta}^{\prime \prime} / \Delta
$$

where $\bar{\tau}_{j}^{\prime}$ and $\bar{\tau}_{j}^{\prime \prime}$ are contained in the interval $\left[\exp (-\Delta / 2) \tau_{j}, \exp (\Delta / 2) \tau_{j}\right]$ and denote the corresponding counterparts of $\tau^{*}$.

The interval $\ln \tau_{j}-\frac{1}{2} \Delta<\ln \tau<\ln \tau_{j}+\frac{1}{2} \Delta$ is the averaging interval over which the mean value is estimated (see Sec. II A earlier).

Because of the reciprocity between the relaxation time $\tau$ and the frequency $\omega$, there is a natural sense in which the length $\Delta$ of the averaging interval, in log-relaxation time, should, in principle at least, correspond to the length $h$ of the sampling interval, in log frequency. However, the problem of determining $H(\tau)$ from either the storage or loss modulus is ill posed. Moreover, the degree of ill posedness in recovering the functional depends on the length of the interval $(\ln a, \ln b)$. The smaller the value of $\Delta$, the more ill posed is the recovery problem. For this reason, it is advantageous to choose the length of $\Delta$ to be larger that $h$, since this improves the stability of the resulting moving-average formulae. Similar techniques have been applied by Anderssen et al. (1998) to construct stable moving-average formulae for the numerical differentiation of observational data.

Suppose, therefore, that $\Delta>h$. Since the averaging procedure discussed above can be centered at each of the relaxation times $\tau_{j}=\omega_{j}^{-1}$, it is convenient to reidentify the mean values given by (31) with the mean values

$$
H\left(\tau_{j}^{\prime}\right)=g_{j, h}^{\prime} / h, \quad \text { and } \quad H\left(\tau_{j}^{\prime}\right)=g_{j, h}^{\prime \prime} / h,
$$

which would have been obtained if the averaging had been performed over averaging intervals of length $\Delta=h$. The values $\tau^{\prime}$ and $\tau^{\prime \prime}$ in (32) now belong to the shorter interval $\ln \tau_{j}-\frac{1}{2} h<\ln \tau<\ln \tau_{j}+\frac{1}{2} h$.

Having estimated the values of $g_{j, \Delta}^{\prime}$ and $g_{j, \Delta}^{\prime \prime}$ on the larger $\Delta$ grid, it is necessary to map them back to the corresponding $g_{j}^{\prime}$ and $g_{j}^{\prime \prime}$ values on the original $h$ grid. The form of the mapping is an immediate consequence of Eqs. (31) and (32), since, for a suitably small $h$,

$$
H\left(\bar{\tau}_{j}^{\prime}\right) \approx H\left(\tau_{j}^{\prime}\right), \quad H\left(\bar{\tau}_{j}^{\prime \prime}\right) \approx H\left(\tau_{j}^{\prime \prime}\right)
$$

One obtains

$$
g_{j}^{\prime}=g_{j, h}^{\prime}=\frac{h}{\Delta} g_{j, \Delta}^{\prime}, \quad \text { and } \quad g_{j}^{\prime \prime}=g_{j, h}^{\prime \prime}=\frac{h}{\Delta} g_{j, \Delta}^{\prime \prime} .
$$

With this rescaling in force, by summing over all the intervals of length $h$, one obtains the following familiar relation for the elastic moduli:

$$
\sum g_{j}^{\prime}=\int_{0}^{\infty} \frac{H(\tau)}{\tau} d \tau .
$$

Finally, specific choices for the averaging interval $\Delta$ and the sampling interval $h$ must be made. As explained earlier, it is advantageous to take $\Delta$ greater than $h$. Since the moving-average formulae to be presented later are intended to be applied to the type of experimental data currently collected on oscillatory shear rheometers, $\Delta$ cannot be greatly 
different from $h$, because of limited availability of data. In this paper, attention is restricted to the choice $\Delta=2 h$.

The actual choice of $h$ must take into account the fact that, for a given $\Delta$, the sample points $l h, l=-L,-L+1, \ldots, L-1, L$, at which the weights $w_{l}^{\prime}(\epsilon)$ and $w_{l}^{\prime \prime}(\epsilon)$ [as defined by Eqs. (29) and (27), respectively], are applied, must satisfy

$$
-\frac{1}{2}(\pi+\Delta) \leqslant l h \leqslant+\frac{1}{2}(\pi+\Delta) .
$$

This implies that, for $\Delta<\pi$,

$$
h \leqslant \frac{\pi}{2(L-1)}, \quad L \geqslant 2 .
$$

The choice $h=\pi /(2 L-1)$ therefore gives the most efficient use of the data for a midpoint rule in that the subintervals for the midpoint rule fit exactly into the interval of integration.

\section{CONSTRUCTION OF THE MOVING-AVERAGE FORMULAE}

As explained in Sec. II B, the construction of the moving-averages for $g_{k}^{\prime}$ and $g_{k}^{\prime \prime}$ reduces to the derivation of the weights $w_{l}^{\prime}(\epsilon)$ and $w_{l}^{\prime \prime}(\epsilon)$ of Eqs. (29) and (27), respectively. This process can be further simplified by introducing the functions

$$
I_{\epsilon}^{\prime}(a)=\int_{0}^{a} E_{\epsilon}^{\prime}(x) d x, \quad I_{\epsilon}^{\prime \prime}(a) \int_{0}^{a} E_{\epsilon}^{\prime \prime}(x) d x, \quad a>0 .
$$

In this way, the evaluation of the weights $w_{l}^{\prime}(\epsilon)$ and $w_{l}^{\prime \prime}(\epsilon)$ reduces to the evaluation of

$$
\begin{aligned}
w_{l}^{\prime}(\epsilon)= & I_{\epsilon}^{\prime}\left[\left(l+\frac{1}{2}\right) h+\frac{1}{2} \Delta\right]-I_{\epsilon}^{\prime}\left[\left(l-\frac{1}{2}\right) h+\frac{1}{2} \Delta\right] \\
& -I_{\epsilon}^{\prime}\left[\left(l+\frac{1}{2}\right) h-\frac{1}{2} \Delta\right]+I_{\epsilon}^{\prime}\left[\left(l-\frac{1}{2}\right) h-\frac{1}{2} \Delta\right], \\
w_{l}^{\prime \prime}(\epsilon)= & I_{\epsilon}^{\prime \prime}\left[\left(l+\frac{1}{2}\right) h+\frac{1}{2} \Delta\right]-I_{\epsilon}^{\prime \prime}\left[\left(l-\frac{1}{2}\right) h+\frac{1}{2} \Delta\right] \\
& -I_{\epsilon}^{\prime \prime}\left[\left(l+\frac{1}{2}\right) h-\frac{1}{2} \Delta\right]+I_{\epsilon}^{\prime \prime}\left[\left(l-\frac{1}{2}\right) h-\frac{1}{2} \Delta\right] .
\end{aligned}
$$

Since $E_{\epsilon}^{\prime}(x)$ and $E_{\epsilon}^{\prime \prime}(x)$ are, respectively, even and odd functions, it follows that:

$$
\begin{gathered}
w_{0}^{\prime}=2\left\{I_{\epsilon}^{\prime}\left[\frac{1}{2}(\Delta+h)\right]-I_{\epsilon}^{\prime}\left[\frac{1}{2}(\Delta-h)\right]\right\}, \\
w_{0}^{\prime \prime}=0 .
\end{gathered}
$$

It is not possible to simply compute the weights by evaluating the functions $I_{\epsilon}^{\prime}(x)$ and $I_{\epsilon}^{\prime \prime}(x)$ numerically for various choices of $\epsilon$ and $x$. In particular, if $0<a<\pi / 2$, then $I_{\epsilon}^{\prime \prime}(a)$ oscillates without bound as $\epsilon \rightarrow 0$; i.e., takes on any positive or negative value an infinite number of times.

In the methodology presented later, explicit use will be made of the following Lemmas.

Lemma 1. For $a \geqslant \frac{1}{2} \pi$,

$$
\lim _{\epsilon \rightarrow 0} I_{\epsilon}^{\prime \prime}(a)=\frac{1}{2}
$$

Proof. It is an immediate consequence of the fact that, as $\epsilon \rightarrow 0$, 


$$
\begin{aligned}
\int_{0}^{a} \operatorname{erf}\left(\frac{x+\frac{1}{2} \pi i}{\sqrt{2} \epsilon}\right) d x \sim & \left(a+\frac{1}{2} \pi i\right)+\sqrt{\frac{2}{\pi}} \frac{\epsilon^{3}}{\left(a+\frac{1}{2} \pi i\right)^{2}} \exp \left[-\frac{\left(a^{2}-\frac{1}{4} \pi^{2}\right)}{2 \epsilon^{2}}\right] \\
& \times \exp \left(-\frac{\pi a i}{2 \epsilon^{2}}\right)+\sqrt{\frac{2}{\pi}} \frac{4 \epsilon^{3}}{\pi^{2}} \exp \left(\frac{\pi^{2}}{8 \epsilon^{2}}\right) .
\end{aligned}
$$

Lemma 2. For arbitrary $\epsilon$ and $l$

$$
w_{-l}^{\prime}(\epsilon)=w_{l}^{\prime}(\epsilon), \quad w_{-l}^{\prime \prime}(\epsilon)=-w_{l}^{\prime \prime}(\epsilon) .
$$

Proof. This is an immediate consequence of the form of the weights $w_{l}^{\prime}(\epsilon)$ and $w_{l}^{\prime \prime}(\epsilon)$ in terms of the even and odd functions $I_{\epsilon}^{\prime}(x)$ and $I_{\epsilon}^{\prime \prime}(x)$, respectively.

The explicit construction of a moving-average formula is explained below for the situation where $h=\pi / 7, \Delta=2 h=2 \pi / 7$ and $L=4$. On the basis of (33), the movingaverage formula (26) becomes

$$
g_{j}^{\prime}(\epsilon)=-\frac{1}{2} \sum_{l=-4}^{4} w_{l}^{\prime \prime}(\epsilon) G^{\prime}\left(\omega_{j+l}\right)
$$

For reasons already mentioned, the limit, as $\epsilon \rightarrow 0$, of each weight in the last equation will not normally exist. However, as will be indicated later, the equation can be given a rigorous meaning if appropriate properties of the complex error function and the definition of the weights $w_{l}^{\prime \prime}(\epsilon)$ are suitably exploited. From Eq. (38), it follows that:

$$
w_{4}^{\prime \prime}(\epsilon)=I_{\epsilon}^{\prime \prime}\left(\frac{11}{14} \pi\right)-I_{\epsilon}^{\prime \prime}\left(\frac{9}{14} \pi\right)-I_{\epsilon}^{\prime \prime}\left(\frac{1}{2} \pi\right)+I_{\epsilon}^{\prime \prime}\left(\frac{5}{14} \pi\right),
$$

and, hence, on invoking Lemma 1, that

$$
w_{4}^{\prime \prime}(\epsilon)=-\frac{1}{2}+I_{\epsilon}^{\prime \prime}\left(\frac{5}{14} \pi\right)+2 \delta_{4},
$$

where $\delta_{4} \rightarrow 0$ as $\epsilon \rightarrow 0$.

Again from (38), it follows that:

$$
\begin{gathered}
w_{3}^{\prime \prime}(\epsilon)=I_{\epsilon}^{\prime \prime}\left(\frac{9}{14} \pi\right)-I_{\epsilon}^{\prime \prime}\left(\frac{1}{2} \pi\right)-I_{\epsilon}^{\prime \prime}\left(\frac{5}{14} \pi\right)+I_{\epsilon}^{\prime \prime}\left(\frac{3}{14} \pi\right), \\
=-I_{\epsilon}^{\prime \prime}\left(\frac{5}{14} \pi\right)+I_{\epsilon}^{\prime \prime}\left(\frac{3}{14} \pi\right)+\delta_{3},
\end{gathered}
$$

where $\delta_{3} \rightarrow 0$ as $\epsilon \rightarrow 0$. Similarly, one finds

$$
w_{2}^{\prime \prime}(\epsilon)=\frac{1}{2}-I_{\epsilon}^{\prime \prime}\left(\frac{5}{14} \pi\right)-I_{\epsilon}^{\prime \prime}\left(\frac{3}{14} \pi\right)+I_{\epsilon}^{\prime \prime}\left(\frac{1}{14} \pi\right)+\delta_{2},
$$

and

$$
w_{1}^{\prime \prime}(\epsilon)=I_{\epsilon}^{\prime \prime}\left(\frac{5}{14} \pi\right)-I_{\epsilon}^{\prime \prime}\left(\frac{3}{14} \pi\right)-2 I_{\epsilon}^{\prime \prime}\left(\frac{1}{14} \pi\right)+\delta_{1},
$$

where $\delta_{2} \rightarrow 0$ and $\delta_{1} \rightarrow 0$ as $\epsilon \rightarrow 0$.

For any given $\epsilon$, we can eliminate the three unknowns $I_{\epsilon}^{\prime \prime}(5 / 14 \pi), I_{\epsilon}^{\prime \prime}(3 / 14 \pi)$, and $I_{\epsilon}^{\prime \prime}(1 / 14 \pi)$ from the four Eqs. (44)-(47). The weights then satisfy the constraint

$$
w_{1}^{\prime \prime}(\epsilon)+2 w_{2}^{\prime \prime}(\epsilon)+3 w_{3}^{\prime \prime}(\epsilon)+4 w_{4}^{\prime \prime}(\epsilon)=-\left(1+\delta_{1}+2 \delta_{2}+3 \delta_{3}+4 \delta_{4}\right) .
$$

Consequently, as $\epsilon \rightarrow 0$, we find 


$$
\sum_{l=1}^{4} l w_{l}^{\prime \prime}(\epsilon) \rightarrow-1
$$

The limit (48) holds even though each weight $w_{l}^{\prime \prime}(\epsilon)$ oscillates without bound as $\epsilon$ $\rightarrow 0$.

The interpretation of the constraint (48) is the key to giving equation (43) a rigorous meaning in the limit $\epsilon \rightarrow 0$. Recall that we are approximating the integral in (8) by product midpoint quadrature. If we choose $G^{\prime}(\omega)$ in (8) to be a quadratic function of $\ln \omega$, i.e., $G^{\prime}(\omega)=A+B \ln \omega+C(\ln \omega)^{2}$ then the integral has the exact value $B \ln (b / a)$. Using (48) it can be easily checked that the formula (43) takes on this precise value in the limit $\epsilon \rightarrow 0$.

This means that there exists a family of moving-average formulae of the form

$$
g_{j}^{\prime}=-\sum_{l=-L}^{L} \alpha_{l} G^{\prime}\left(\omega_{j+l}\right),
$$

with

$$
\begin{gathered}
\alpha_{-l}=-\alpha_{l}, \quad \alpha_{0}=0, \\
\sum_{l=1}^{L} l \alpha_{l}=-\frac{1}{2},
\end{gathered}
$$

such that each formula in this family evaluates the integral in (8) exactly whenever $G^{\prime}(\omega)$ is quadratic in $\ln \omega$ in the interval

$$
-\left(L+\frac{1}{2}\right) h \leqslant \ln \left(\frac{\omega}{\omega_{j}}\right) \leqslant\left(L+\frac{1}{2}\right) h .
$$

Although (49) and (51) have been derived for the case $L=4$, they hold true for all $L \geqslant 2$. The constraints (50) and (51) are the only constraints satisfied by the $\alpha$ coefficients of formula (49). They are insufficient to determine the coefficients themselves.

For the analysis of the numerical performance of formula (49), one can exploit the fact that $g_{j}^{\prime}$ is simply a linear combination of the same difference $G^{\prime}\left(\omega_{j+l}\right)-G^{\prime}\left(\omega_{j-l}\right)$ on larger and larger grids (i.e., with $l=1,2, \ldots$ ); namely,

$$
g_{j}^{\prime}=-\sum_{l=1}^{L} \alpha_{l}\left[G^{\prime}\left(\omega_{j+l}\right)-G^{\prime}\left(\omega_{j-l}\right)\right] .
$$

For such an analysis, one can exploit the methodology developed by Anderssen et al. (1998) for the analysis of moving-average (finite difference) differentiators.

We now discuss the moving average-formula for loss moduli. On the basis of (33), the formula (28) becomes, when $h=\pi /(2 L-1)$,

$$
g_{j}^{\prime \prime}(\epsilon)=\frac{1}{2} \sum_{l=-L}^{L} w_{l}^{\prime}(\epsilon) G^{\prime \prime}\left(\omega_{j+l}\right) .
$$

A similar procedure to that used in deriving (49) from (43) may be used to derive a family of moving-average formulae of the form

$$
g_{j}^{\prime \prime}=\sum_{l=-L}^{L} \beta_{l} G^{\prime \prime}\left(\omega_{j+l}\right),
$$


from (54). The coefficients $\beta_{l}$ satisfy the conditions

$$
\beta_{-l}=\beta_{l}, \quad \sum_{l=1, l \text { odd }}^{L} \beta_{l}=\frac{h}{2 \pi}, \quad \frac{1}{2} \beta_{0}+\sum_{l=2, l \text { even }}^{L} \beta_{l}=\frac{h}{2 \pi} .
$$

These conditions are sufficient to guarantee that formula (55) is exact whenever $G^{\prime \prime}(\omega)$ is linear in $\ln \omega$ in the interval (52), but are insufficient to determine the coefficients themselves.

For the analysis of the numerical performance of formula (55), one can exploit the fact that $g_{j}^{\prime \prime}$ is simply a linear combination of the same sums $G^{\prime \prime}\left(\omega_{j+l}\right)+G^{\prime \prime}\left(\omega_{j-l}\right)$ on larger and larger grids (i.e., with $l=1,2, \ldots$ ); namely,

$$
g_{j}^{\prime \prime}=\beta_{0} G^{\prime \prime}\left(\omega_{j}\right)+\sum_{l=1}^{L} \beta_{l}\left[G^{\prime \prime}\left(\omega_{j-l}\right)+G^{\prime \prime}\left(\omega_{j+l}\right)\right] .
$$

One can therefore anticipate that, because the $\beta$ moving-average has this summation interpretation, it will behave in a more stable manner than the $\alpha$-moving average (when applied to data with the same noise levels). On the other hand, one would expect that the $\alpha$-moving average, when it performs well because the level of the noise on the data is suitably small, will give a better resolution of the relaxation spectrum than the $\beta$-moving average. These tentative conclusions will be verified for noisy synthetic data in the sequel.

It has already been stated that the constraints (50)-(51) and (56) are insufficient to determine the coefficients. Indeed, the constraints alone impart to the formulae only a very basic accuracy, as we have seen. For practical purposes it is necessary to improve on this accuracy, and this can be done by optimizing the choice of coefficients. There is no unique way of doing this. The strategy proposed in this paper is to maximize the resolving power of each formula (see Sec. IV C). The approach is the same for both the $\alpha$ and the $\beta$ coefficients. For the $\alpha$ coefficients, the evaluation reduces to the following steps:

(1) Choose as a representative basis function for $H(\tau)$, the delta-function $\delta\left(\tau-\tau_{0}\right)$ centered at an arbitrary $\tau_{0}>0$, for which the corresponding values of $G^{\prime}(\omega)$ and $G^{\prime \prime}(\omega)$ can be determined analytically. In particular, if one chooses

$$
H(\tau)=\delta(\tau-1)
$$

the corresponding values of $G^{\prime}(\omega)$ and $G^{\prime \prime}(\omega)$, as defined by Eqs. (5) and (6), become

$$
G^{\prime}(\omega)=\frac{\omega^{2}}{1+\omega^{2}}, \quad G^{\prime \prime}(\omega)=\frac{\omega}{1+\omega^{2}}
$$

(2) In Eqs. (1) and (2), replace $G_{N}^{\prime}(\omega)$ and $G_{N}^{\prime \prime}(\omega)$ by the corresponding analytic expressions for $G^{\prime}(\omega)$ and $G^{\prime \prime}(\omega)$, and replace the $g_{j}$ by the expression (16) for $g_{j}^{\prime}$. For the choice (58), one thereby obtains the expressions

$$
G^{\prime}\left(\omega_{k}\right)=\frac{\omega_{k}^{2}}{1+\omega_{k}^{2}}=-\sum_{j=L}^{K-L} \sum_{l=-L}^{L} \alpha_{l} G^{\prime}\left(\omega_{j+l}\right) \frac{\omega_{k}^{2} \tau_{j}^{2}}{1+\omega_{k}^{2} \tau_{j}^{2}},
$$

and

$$
G^{\prime \prime}\left(\omega_{k}\right)=\frac{\omega_{k}}{1+\omega_{k}^{2}}=-\sum_{j=L}^{K-L} \sum_{l=-L}^{L} \alpha_{l} G^{\prime}\left(\omega_{j+l}\right) \frac{\omega_{k} \tau_{j}}{1+\omega_{k}^{2} \tau_{j}^{2}},
$$

where $k$ ranges over the set of integers which satisfy $0 \leqslant k \leqslant K$, and $\tau_{j}=1 / \omega_{j}$. 
TABLE I. The $M A\left[L, G^{\prime}\right]$ moving-average formulae.

\begin{tabular}{|c|c|c|c|c|c|c|c|}
\hline & & & $g_{j}^{\prime}=-\Sigma_{l=}^{L}$ & ${ }_{l} G^{\prime}\left(\omega_{j+l}\right.$ & $\alpha_{-l}=-\alpha_{l}$ & & \\
\hline$L$ & $h$ & $\alpha_{0}$ & $\alpha_{1}$ & $\alpha_{2}$ & $\alpha_{3}$ & $\alpha_{4}$ & $\nu_{\alpha}$ \\
\hline 2 & $\frac{\pi}{3}$ & 0.0 & -0.74984822 & 0.12492411 & & & 1.08 \\
\hline 3 & $\frac{\pi}{5}$ & 0.0 & -2.36670401 & 1.17865243 & -0.16353362 & & 3.75 \\
\hline 4 & $\frac{\pi}{7}$ & 0.0 & -10.88578711 & 8.49765772 & -2.60305279 & 0.29990751 & 19.88 \\
\hline
\end{tabular}

(3) Apply weighted least squares to solve the overdetermined systems of equations constructed in Step 2 along with the constraints

$$
\alpha_{0}=0, \quad \alpha_{-l}=-\alpha_{l}, \quad l=1,2, \ldots, L, \quad \sum_{l=1}^{L} l \alpha_{l}=-\frac{1}{2},
$$

For the choice of the basis function being examined, this reduces to finding the values of the $\alpha_{l}$ which minimize

$$
\begin{aligned}
\sum_{k=0}^{K}[1+ & \left.\frac{1}{G^{\prime}\left(\omega_{k}\right)} \sum_{j=L}^{K-L} \sum_{l=-L}^{L} \alpha_{l} G^{\prime}\left(\omega_{j+l}\right) \frac{\omega_{k}^{2} \tau_{j}^{2}}{1+\omega_{k}^{2} \tau_{j}^{2}}\right]^{2} \\
& +\sum_{k=0}^{K}\left[1+\frac{1}{G^{\prime \prime}\left(\omega_{k}\right)} \sum_{j=L}^{K-L} \sum_{l=-L}^{L} \alpha_{l} G^{\prime}\left(\omega_{j+l}\right) \frac{\omega_{k} \tau_{j}}{1+\omega_{k}^{2} \tau_{j}^{2}}\right]^{2},
\end{aligned}
$$

subject to the constraints $(62)$.

The size of the noise amplification factor $\nu_{\alpha}$, which is defined by

$$
\nu_{\alpha}=\sqrt{\sum_{l=-L}^{L} \alpha_{l}^{2}}
$$

is a measure of the stability of the resulting moving-average formula which the $\alpha_{l}$ generate.

A similar argument applies for the determination of the $\beta_{l}$ coefficients. The corresponding noise amplification factor $\nu_{\beta}$ is defined by

$$
\nu_{\beta}=\sqrt{\sum_{l=-L}^{L} \beta_{l}^{2}}
$$

For the delta basis function examined before, Tables I and II list, respectively, the values of $\alpha_{l}$ and $\beta_{l}$ for $L=2,3,4$, along with the corresponding values of the amplifaction factors $\nu_{\alpha}$ and $\nu_{\beta}$. Though basis functions other than the delta-function $\delta(\tau$ -1 ) could have been invoked to estimate appropriate values for the $\alpha_{l}$ and $\beta_{l}$, they are not pursued in this paper. Since any spectrum $H(\tau)$ can be approximated by a linear combination of basis functions of the form $\delta\left[\ln \left(\tau / \tau_{j}\right)\right]$, which correspond to various translations of the single-mode delta-function spectrum, and since the formulae (16) and (17), as well as (49) and (55), are also translationally invariant, it follows that the 
TABLE II. The $M A\left[L, G^{\prime \prime}\right]$ moving-average formulae.

\begin{tabular}{cccccccc}
\hline \hline \multicolumn{7}{c}{$g_{j}^{\prime \prime}=\Sigma_{l=-L}^{L} \beta_{l} G^{\prime \prime}\left(\omega_{j+l}\right)}$, & $\beta_{-l}=\beta_{l}$ \\
\hline$L$ & $h$ & $\beta_{0}$ & $\beta_{1}$ & $\beta_{2}$ & $\beta_{3}$ & $\beta_{4}$ & $\nu_{\beta}$ \\
2 & $\frac{\pi}{3}$ & 0.65068623 & $1 / 6$ & -0.15867645 & & & 0.73 \\
& & & & & \\
& $\frac{\pi}{5}$ & 1.49882489 & -0.07096359 & -0.64941244 & 0.17096359 & & \\
4 & $\frac{\pi}{7}$ & 6.20451011 & -1.67024098 & -2.73004184 & 1.74166954 & -0.30078464 & 8.08 \\
\hline \hline
\end{tabular}

moving-averages tabulated in Tables I and II are able to recover such a basis function representation for an arbitrary spectrum with the same accuracy with which they recover a single-mode spectrum.

Notation. In the sequel, use will be made of the following notation: the movingaverage formulae of length $2 L+1$ constructed from the $\alpha_{l}$ will be denoted by $M A\left[L, G^{\prime}\right]$, whereas the corresponding $\beta_{l}$ formulae will be denoted by $M A\left[L, G^{\prime \prime}\right]$. The parameter $L$ can be viewed as the independent variable of these formulae as it determines both the length $2 L+1$ of these moving-averages and the sampling interval $h=\pi /(2 L$ $-1)$ of the grid on which they are applied.

It is important to note that, as the sampling interval $\pi /(2 L-1)$ for each movingaverage formula decreases, the corresponding noise amplification factor (either $\nu_{\alpha}$ or $\nu_{\beta}$ ) increases. The noise amplification factor is a direct measure of the degree of ill posedness for recovering the spectrum from sampled oscillatory shear data. The smaller the sampling interval, the greater is the resolution to be expected when recovering the spectrum from exact data. On the other hand, when working with noisy data, the greater the amplification of the noise in these data. In practice, the correct balance between high resolution and low noise amplification must be found. In the sequel, an algorithm is proposed which involves the dual application of the $\alpha$ and $\beta$ moving-average formulae to the $G^{\prime \prime}(\omega)$ data.

\section{NUMERICAL IMPLEMENTATION AND VALIDATION}

\section{A. Introduction}

When the storage and loss moduli $G^{\prime}(\omega)$ and $G^{\prime \prime}(\omega)$, are sampled at $K+1$ frequencies $\omega_{k}=\omega_{0} \exp (k h), k=0, \ldots, K$, with sampling interval $h=\pi /(2 L-1)$, the application of the moving average formulae of Tables I and II will generate estimates of the discrete elastic moduli $g_{j}^{\prime}$ and $g_{j}^{\prime \prime}$ corresponding to the relaxation times $\tau_{j}=\omega_{j}^{-1}$, $j=L, \ldots, K-L$. As explained in Sec. II C, point estimates of the continuous relaxation spectrum are then given by

$$
H_{R}\left(\tau_{j}\right)=\frac{1}{h} g_{j}^{\prime} \quad \text { or } \quad H_{R}\left(\tau_{j}\right)=\frac{1}{h} g_{j}^{\prime \prime},
$$

where $H_{R}(\tau)$ denotes the recovered spectrum. In addition, the discrete elastic moduli $g_{j}^{\prime}$ and $g_{j}^{\prime \prime}$ can be used to reconstruct the following continuous estimates of the storage and loss moduli: 


$$
G_{R}^{\prime}(\omega)=\sum_{j=L}^{K-L} g_{j}^{\prime} \frac{\omega^{2} \tau_{j}^{2}}{1+\omega^{2} \tau_{j}^{2}}, \quad G_{R}^{\prime \prime}(\omega)=\sum_{j=L}^{K-L} g_{j}^{\prime} \frac{\omega \tau_{j}}{1+\omega^{2} \tau_{j}^{2}},
$$

and

$$
G_{R}^{\prime}(\omega)=\sum_{j=L}^{K-L} g_{j}^{\prime \prime} \frac{\omega^{2} \tau_{j}^{2}}{1+\omega^{2} \tau_{j}^{2}}, \quad G_{R}^{\prime \prime}(\omega)=\sum_{j=L}^{K-L} g_{j}^{\prime \prime} \frac{\omega \tau_{j}}{1+\omega^{2} \tau_{j}^{2}} .
$$

These reconstructions $G_{R}^{\prime}(\omega)$ and $G_{R}^{\prime \prime}(\omega)$ of the storage and loss moduli can be compared with the actual sampled data $G^{\prime}\left(\omega_{k}\right)$ and $G^{\prime \prime}\left(\omega_{k}\right)$.

Because the recovery of the relaxation spectrum is ill posed, a good fit of the reconstructions to the sampled storage and loss moduli measurements is not a sufficient condition for an accurate recovery of the spectrum. (It is, however, a necessary condition.) This crucial fact is often overlooked in situations where the underlying nature and consequences of the ill posedness of the reconstruction problem are not fully understood.

\section{B. Recovering the delta-function from exact data}

For a single-mode relaxation spectrum

$$
H(\tau)=\delta(\tau-1)=\delta(\ln \tau),
$$

the moving-average formulae $M A\left[L, G^{\prime}\right]$ and $M A\left[L, G^{\prime \prime}\right]$ not only recover estimates of the elastic moduli but also the following continuous approximations, respectively, to the delta-function:

$$
H_{R}(\tau)=-\frac{1}{h} \sum_{l=-L}^{L} \alpha_{l} G^{\prime}[\exp (l h) / \tau]
$$

and

$$
H_{R}(\tau)=\frac{1}{h} \sum_{l=-L}^{L} \beta_{l} G^{\prime \prime}[\exp (l h) / \tau]
$$

where $G^{\prime}(\omega)$ and $G^{\prime \prime}(\omega)$ take the forms given in (61).

The exact oscillatory shear data were generated by evaluating $G^{\prime}(\omega)$ and $G^{\prime \prime}(\omega)$, given in Eq. (61), on four grids of frequencies given by $\omega_{k}=\omega_{0} \exp (k h), k=0, \ldots, K$, with sampling interval $h=\pi /(2 L-1)$. The values of $K$ were $12,20,28$, and 36, while the values of $L$ were $2,3,4$, and 5 . In each case, $\omega_{0}=\exp (-2 \pi)$.

The results obtained, when the moving-average formulae $M A\left[L, G^{\prime}\right]$ and $M A\left[L, G^{\prime \prime}\right], L=2,3,4,5$, are applied to the exact storage and loss moduli, are plotted in Figs. 1(a) and 2(a), respectively. It is clear from these plots that

(i) Both the $M A\left[L, G^{\prime}\right]$ and $M A\left[L, G^{\prime \prime}\right]$ formulae give stable recoveries of the deltafunction even when the value of $L$ is small.

(ii) Increasingly better and better recovery of the delta-function is obtained with both formulae as the value of $L$ increases. In addition, as Figs. 1(b) and 2(b) show, when $L$ $=4$, the reconstructed data functions $G_{R}^{\prime}(\omega)$ and $G_{R}^{\prime \prime}(\omega)$, agree to graphical accuracy with the values of $G^{\prime}(\omega)$ and $G^{\prime \prime}(\omega)$.

(iii) The recovery obtained from the $M A\left[L, G^{\prime}\right]$ formulae, $L=2,3,4,5$, are clearly sharper and better resolved than the corresponding recovery obtained from the $M A\left[L, G^{\prime \prime}\right]$ formulae. This observation can be explained in terms of the numerical performance of the $M A\left[L, G^{\prime}\right]$ and $M A\left[L, G^{\prime \prime}\right]$ formulae. Because they correspond to com- 

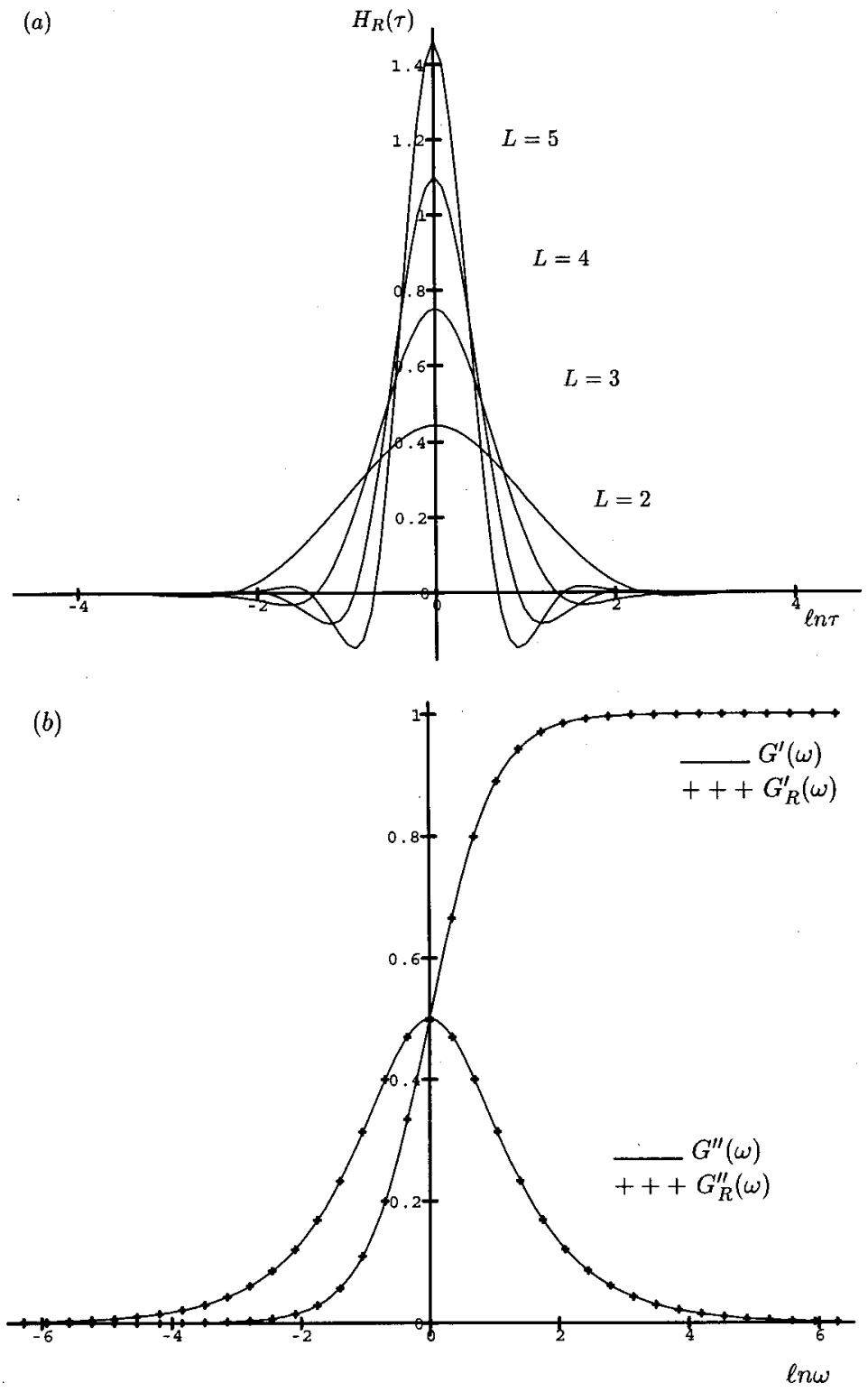

FIG. 1. (a) Plots of $H_{R}(\tau)$ vs $\ln \tau$, given by (4.5), with $L=2,3,4,5$. Basewidth of each curve indicates the resolving power of the formula. (b) Reconstructions of the storage and loss moduli $G_{R}^{\prime}(\omega)$ and $G_{R}^{\prime \prime}(\omega)$ given by (4.2) when $L=4$.

bining differences on larger and larger grids [cf. (53)], the $M A\left[L, G^{\prime}\right]$ formulae behave "notionally" like numerical differentiators in a derivative spectroscopy context [cf. O'Haver (1997)], and therefore will tend to sharpen the higher frequency components in a reconstruction. On the other hand, because the $M A\left[L, G^{\prime \prime}\right]$ formulae correspond to combining summations on larger and larger grids [cf. (58)], they behave more like smoothers, although the alternating signs of the coefficients also contribute a sharpening contribution. This essential difference will be explicitly exploited in the duality algorithm to be proposed later. 

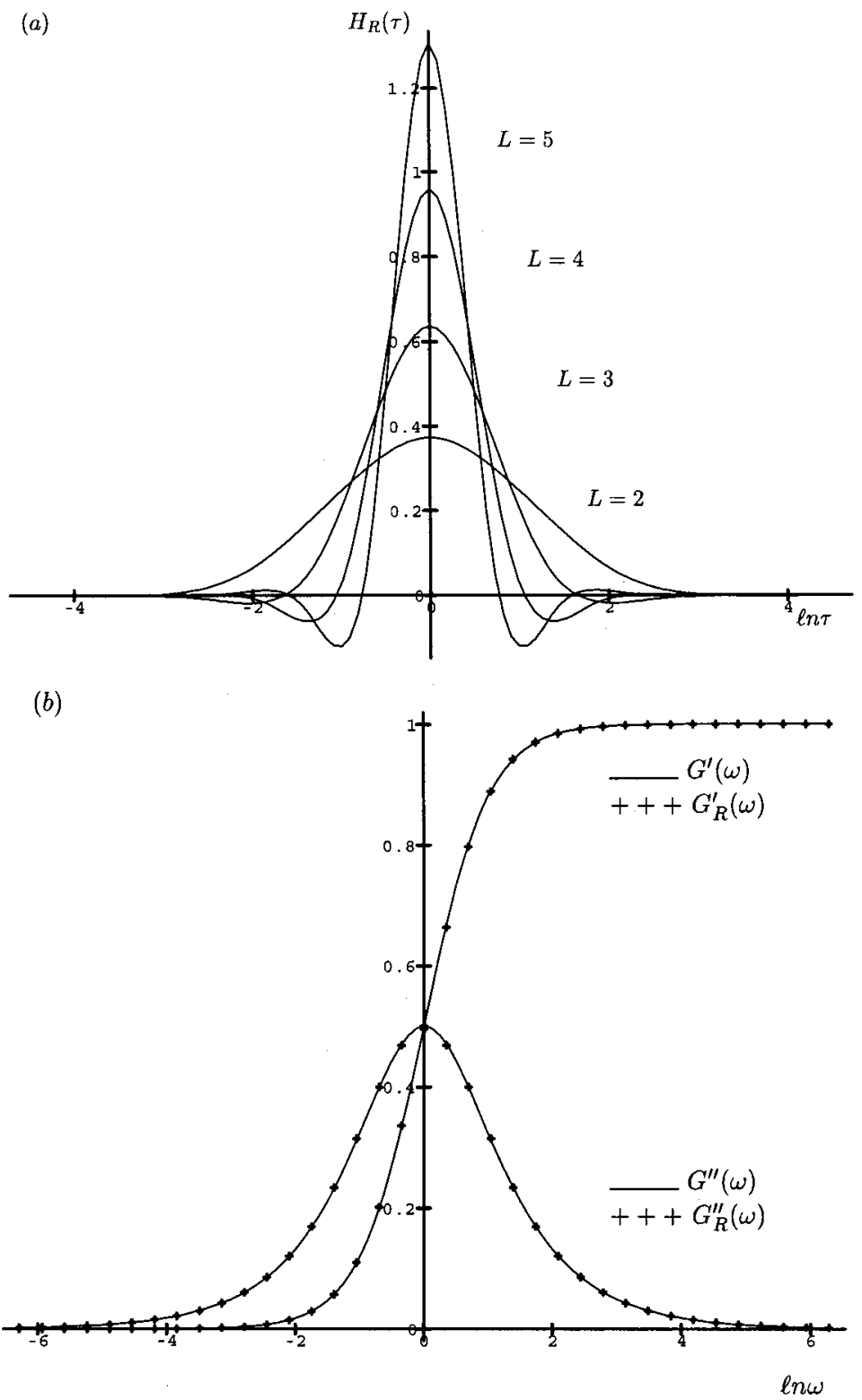

FIG. 2. (a) Plots of $H_{R}(\tau)$ vs $\ln \tau$, given by (4.6), with $L=2,3,4$, 5. Basewidth of each curve indicates the resolving power of the formula. (b) Reconstructions of the storage and loss moduli $G_{R}^{\prime}(\omega)$ and $G_{R}^{\prime \prime}(\omega)$ given by (4.3) when $L=4$.

\section{Resolving power}

In recovering the relaxation spectrum using moving-average formulae, temporal resolution is limited by the finite sampling interval of the data. This is evident from Figs. 1(a) and 2(a) which show that a delta function is recoverable as a broadened pulse, the breadth of which decreases as the sampling interval becomes smaller. Calculating the maximum possible temporal resolution requires an arbitrary definition of what is meant by resolving two features. A working definition of resolving power is given later. 
Consider a relaxation spectrum consisting of two delta-functions centered at $\tau=\tau_{0}$ and $\tau=\tau_{0}^{-1}$, respectively; i.e.,

$$
H(\tau)=\delta\left(\tau-\tau_{0}\right)+\delta\left(\tau-\tau_{0}^{-1}\right) .
$$

The separation between these two peaks, measured in decades, is given by

$$
\mu\left(\tau_{0}\right)=2 \log _{10}\left(\tau_{0}\right) .
$$

The $M A\left[L, G^{\prime}\right]$ moving-average formula recovers the continuous approximation to the spectrum given by

$$
H_{R}(\tau)=-\frac{1}{h} \sum_{l=L}^{L} \alpha_{l} G^{\prime}[\exp (l h) / \tau]
$$

where

$$
G^{\prime}(\omega)=\frac{\omega^{2} \tau_{0}^{2}}{1+\omega^{2} \tau_{0}^{2}}+\frac{\omega^{2} \tau_{0}^{-2}}{1+\omega^{2} \tau_{0}^{-2}} .
$$

If $\tau_{0}$ is not too small, the graph of $H_{R}(\tau)$ will consist of two broadened pulses of separation $\mu$, with a central minimum of height $H_{R}(1)$. As $\tau_{0}$ is increased, the height of the central minimum $H_{R}(1)$ decreases until it reaches zero, at which point the two pulses are completely resolved. If $\tau_{\max }>0$ is the smallest value of $\tau_{0}$ for which $H_{R}(1)$ $=0$, the corresponding value of $\mu\left(\tau_{\max }\right)$ is defined to be the least completely resolvable separation (LCRS). All separations with $\tau_{0}>\tau_{\max }$ will also be completely resolvable. Figure 3(a) shows the graph of $H_{R}(\tau)$ recovered from the formula $M A\left[4, G^{\prime}\right]$, where $\tau_{0}=\tau_{\max }=2.5 \mathrm{~s}$. The LCRS $\mu\left(\tau_{\max }\right)$ takes the value of 0.8 decades.

On the other hand, if $\tau_{0}$ is decreased, the height of the central minimum $H_{R}(1)$ will grow until it is no longer a central minimum, at which point the two pulses appear as a single, completely unresolved pulse. If $\tau_{\min }>0$ is the greatest value of $\tau_{0}$ for which $H_{R}(1)$ is not a minimum, and $\tau_{\min }<\tau_{\max }$, the corresponding value of $\mu\left(\tau_{\min }\right)$ is defined to be the greatest completely unresolvable separation (GCUS). All separations with $\tau_{0}<\tau_{\min }$ will also be completely unresolvable. Figure 3(b) shows the graph of $H_{R}(\tau)$ recovered from the formula $M A\left[4, G^{\prime}\right]$, where $\tau_{0}=\tau_{\min }=1.46 \mathrm{~s}$. The GCUS $\mu\left(\tau_{\text {max }}\right)$ takes the value of 0.33 decades.

The LCRS and GCUS represent two extreme situations, that of complete resolution and that of no resolution, respectively. In practice, values of $\tau_{0}$ between the two extreme values $\tau_{\min }$ and $\tau_{\max }$ will result in partially resolved peaks. The concept of a mean resolvable separation (MRS) is introduced and defined to be the separation $\mu$ for which

$$
\tau_{0}=\tau_{\text {mean }} \equiv \sqrt{\tau_{\min } \tau_{\max }}
$$

the geometric mean. Figure 3(c) shows the graph of $H_{R}(\tau)$ recovered by formula $M A\left[4, G^{\prime}\right]$, when $\tau_{0}=\tau_{\text {mean }}=1.91 \mathrm{~s}$. The MRS $\mu\left(\tau_{\text {mean }}\right)$ takes the value of 0.56 decades. This is a realistic expectation of resolvable separation from this nine-point formula. Tables III and IV give the LCRS, GCUS, and MRS values for the six movingaverage formulae which appear in Tables I and II.

It is clear from these tables that the MRS decreases with $h$, the sampling interval. In addition, the resolving power of the $\beta$ formulae are not as great as for the $\alpha$ formulae, for the reasons explained above. The MRS values given in the tables should be used to assess whether peaks which appear in the recovered spectrum are real or spurious (i.e., resulting from noise amplification). 

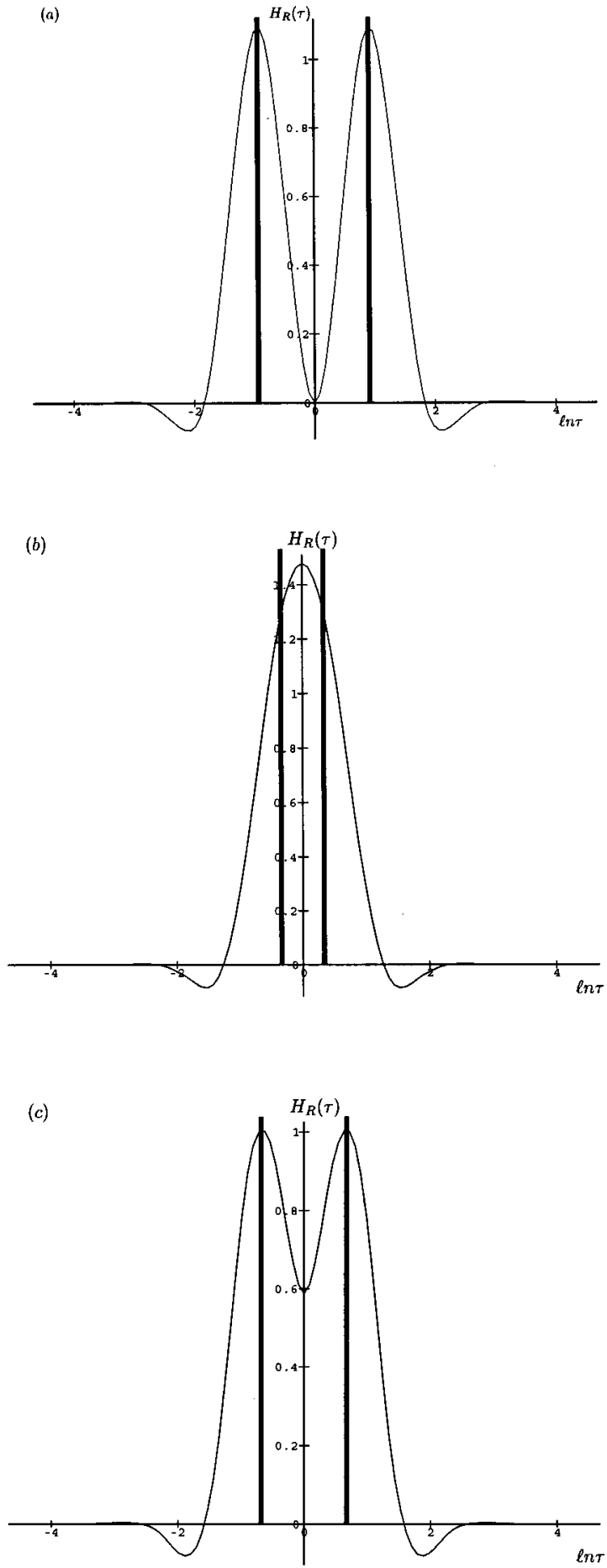

FIG. 3. Plots of $H_{R}(\tau)$ vs $\ln \tau$, given by (4.9) with $L=4$, for two delta functions with separation $2 \ln \tau_{0}$. (a) Shows the least completely resolvable separation for $L=4\left(\tau_{0}=2.5\right)$. (b) Shows the greatest completely unresolvable separation for $L=4\left(\tau_{0}=1.46\right)$. (c) Shows the mean resolvable separation for $L=4$ $\left(\tau_{0}=1.91\right)$. 
TABLE III. LCRS, GCUS, and MRS values for the moving-average formulae of Table I.

\begin{tabular}{cccc}
\hline \hline Formula & $\begin{array}{c}\text { LRCS } \\
\text { (decades) }\end{array}$ & $\begin{array}{c}\text { GCUS } \\
\text { (decades) }\end{array}$ & $\begin{array}{c}\text { MRS } \\
\text { (decades) }\end{array}$ \\
\hline$M A\left[2, G^{\prime}\right]$ & 2.16 & 0.60 & 1.38 \\
$M A\left[3, G^{\prime}\right]$ & 1.18 & 0.42 & 0.80 \\
$M A\left[4, G^{\prime}\right]$ & 0.80 & 0.33 & 0.56 \\
\hline \hline
\end{tabular}

\section{Noise and measurement errors}

In order to simulate the measurement errors in $G^{\prime}(\omega)$ and $G^{\prime \prime}(\omega)$ in a realistic manner, it is necessary to allow for the fact that these quantities have been computed from the amplitude ratio $\vartheta$ and the phase lag $c$, obtained from a conventional oscillatory test, using the following formulae:

$$
G^{\prime}(\omega)=\frac{\omega S \vartheta(\cos c-\vartheta)}{\vartheta^{2}-2 \vartheta \cos c+1},
$$

and

$$
G^{\prime \prime}(\omega)=-\frac{\omega S \vartheta \sin c}{\vartheta^{2}-2 \vartheta \cos c+1},
$$

where $S$ corresponds to a geometric parameter of the form

$$
S=\frac{k}{\omega}\left(\Omega^{2}-\omega^{2}\right),
$$

with $k$ and $\Omega$ denoting constants which depend on the rheogoniometer being used. In particular, $\Omega$ corresponds to the natural frequency of the instrument, a representative value of which is taken to be $4 \pi \mathrm{rad} / \mathrm{s}$. The value of $k$ is chosen to be $0.02 \eta_{0}$, where $\eta_{0}$ is the zero-shear-rate viscosity of the material being tested.

Given an exact relaxation spectrum $H(\tau)$, one first calculates the exact values for $G^{\prime}(\omega)$ and $G^{\prime \prime}(\omega)$ from (5) and (6), and hence the exact complex viscosity $\eta^{*}(\omega)$ $=\omega^{-1}\left[G^{\prime}(\omega)-i G^{\prime \prime}(\omega)\right]$. Next, the exact amplitude ratio $\vartheta$ and phase lag $c$ are calculated, as a function of frequency $\omega$, from the formula [Walters (1987), p. 127]

$$
\frac{\exp [i c(\omega)]}{\vartheta(\omega)}=1-\frac{i}{\eta^{*}(\omega)} S .
$$

Random Gaussian noise is then added to the $\vartheta\left(\omega_{k}\right)$ and $c\left(\omega_{k}\right)$ in proportion to their magnitudes. For the $\vartheta\left(\omega_{k}\right)$, a standard deviation of $3 \%$ is chosen, while, for $c\left(\omega_{k}\right)$, the standard deviation is $1 \%$. The required noisy storage and loss moduli are then computed

TABLE IV. LCRS, GCUS, and MRS values for the moving-average formulae of Table II.

\begin{tabular}{cccc}
\hline \hline Formula & $\begin{array}{c}\text { LRCS } \\
\text { (decades) }\end{array}$ & $\begin{array}{c}\text { GCUS } \\
\text { (decades) }\end{array}$ & $\begin{array}{c}\text { MRS } \\
\text { (decades) }\end{array}$ \\
\hline$M A\left[2, G^{\prime \prime}\right]$ & 2.76 & 0.54 & 1.65 \\
$M A\left[3, G^{\prime \prime}\right]$ & 1.43 & 0.45 & 0.94 \\
$M A\left[4, G^{\prime \prime}\right]$ & 0.92 & 0.36 & 0.64 \\
\hline \hline
\end{tabular}


from (77) and (78). It is important to note that the resulting noise on $G^{\prime}\left(\omega_{k}\right)$ and $G^{\prime \prime}\left(\omega_{k}\right)$ is not proportional to their magnitudes, particularly at high frequencies.

Where necessary, allowances will be made for natural frequency errors in $G^{\prime}\left(\omega_{k}\right)$ and $G^{\prime \prime}\left(\omega_{k}\right)$ resulting from the use of (77), (78), and (79). Here, use is made of the interpolation procedure proposed by Walters (1987), pp. 151-155. No account is taken of other possible errors.

\section{E. Recovering the delta function from noisy data}

Using the $G^{\prime}(\omega)$ and $G^{\prime \prime}(\omega)$ of (59), the noisy (inexact) oscillatory shear data were generated by applying the procedure outlined in Sec. IV D. The noisy data are shown in Figs. 4(b), 4(d), and 4(f) for sampling intervals $h$ corresponding to $L=2,3,4$.

The results obtained, when the moving-average formulae $M A\left[L, G^{\prime \prime}\right]$ are applied to the noisy data, are plotted in Figs. 4(a), 4(c), and 4(e). It is clear from these plots that:

(i) The amplification of the noise in the loss modulus by the $M A\left[L, G^{\prime \prime}\right]$ formulae is not a problem when $L=2$ and $L=3$. However, when $L=4$, the noise in the data is amplified to give several spurious peaks, the largest of which occurs at the low relaxation time of $\tau=\exp (-4)$, and results from the amplification of high frequency noise.

Next, the effect of reduced noise levels in the data is considered. Two new data sets were generated for a sampling interval corresponding to $L=4$. The first had random Gaussian noise with a standard devation of $1.5 \%$ in the amplitude ratio $\vartheta$, and $0.5 \%$ in the phase lag $c$. The results obtained with the $M A\left[4, G^{\prime \prime}\right]$ formula are shown in Fig. 5(a), with the data and corresponding reconstructions in Fig. 5(b). The second data set had noise with a standard devation of $0.6 \%$ in $\vartheta$ and $0.2 \%$ in $c$. The results obtained with the $M A\left[4, G^{\prime \prime}\right]$ formula are shown in Fig. 5(c), with the data and corresponding reconstructions in Fig. 5(d). It is clear that:

(ii) As the noise level decreases, the recovery of the spectrum from $M A\left[4, G^{\prime \prime}\right]$ approaches the spectrum recovered from exact data.

In this way, one obtains the following very important conclusions about the numerical performance of the $M A\left[L, G^{\prime}\right]$ and $M A\left[L, G^{\prime \prime}\right]$ formulae.

Conclusion 4.1. For a given $L$, there is an upper level to the noise on the data such that below this level the moving-average formulae give good recoveries of the spectrum to within the resolving power of the formulae.

Conclusion 4.2. With respect to the noisy data, the moving-average formulae $M A\left[L, G^{\prime}\right]$ and $M A\left[L, G^{\prime \prime}\right]$ are "asymptotically-stable" in that, as the level of noise on the data decreases, one obtains a closer and closer agreement with the reconstructions obtained using exact data.

Clearly, the results obtained by $M A\left[4, G^{\prime \prime}\right]$ in Fig. 4(e) are far from satisfactory. The resulting reconstructions $G_{R}^{\prime}$ and $G_{R}^{\prime \prime}$ in Fig. 4(f), however, provide good smoothing curves for the noisy data. In what follows we exploit this result in an iterative algorithm which improves the performance of both sets of formulae.

\section{F. A duality algorithm}

So far, it has been demonstrated that the moving average formulae $M A\left[L, G^{\prime}\right]$ and $M A\left[L, G^{\prime \prime}\right]$ are capable of recovering delta functions, to within finite resolution dependent on $L$, provided the noise levels in the storage and loss moduli are not too high. Furthermore, the noise amplification factor for the formula $M A\left[L, G^{\prime \prime}\right]$ is less than that for $M A\left[L, G^{\prime}\right]$, while the resolving power of $M A\left[L, G^{\prime}\right]$ is greater than that of 


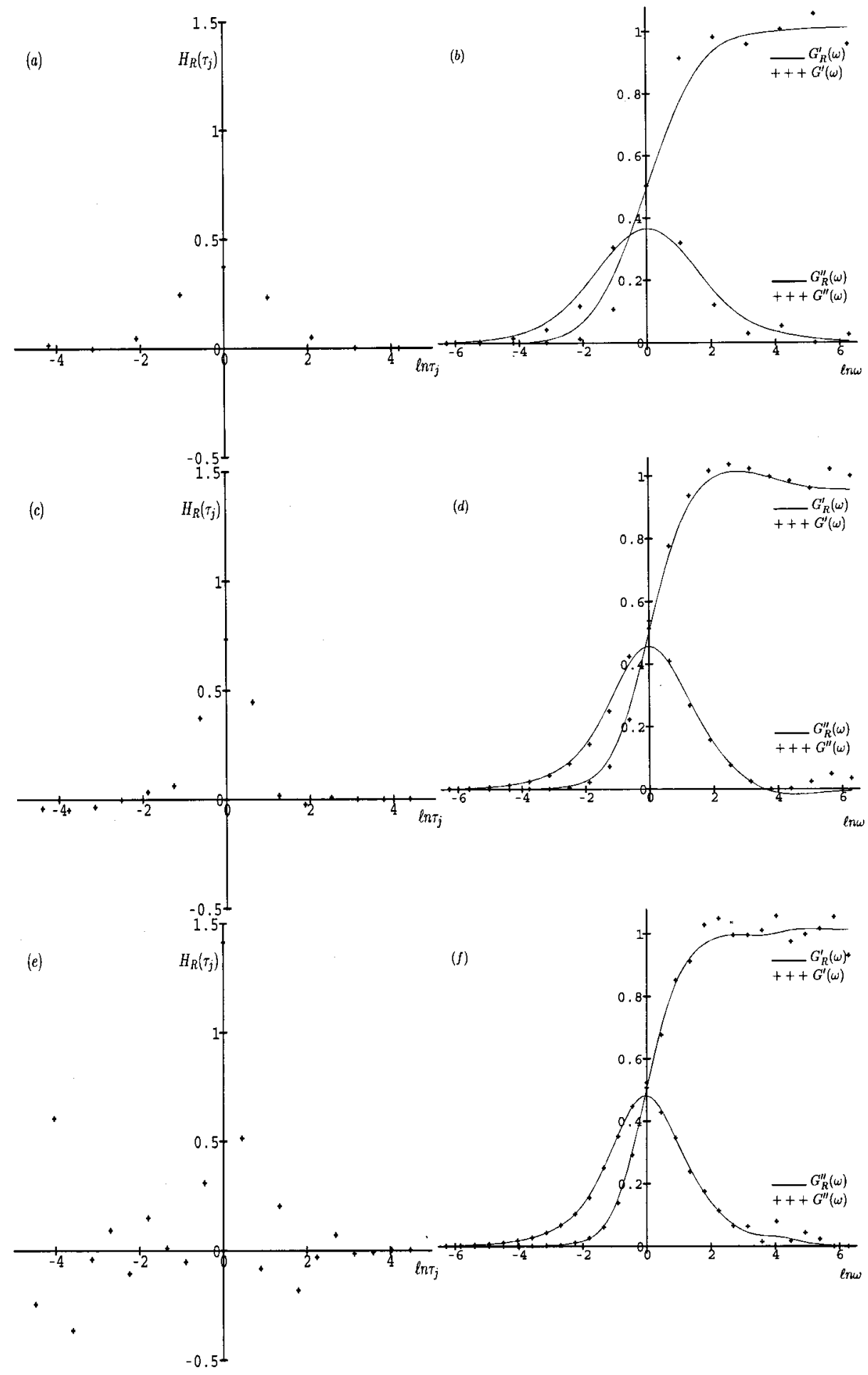

FIG. 4. Left column shows recovery of the delta function from a noisy loss modulus (1\% noise in phase lag, $3 \%$ noise in amplitude ratio). (a) Using $M A\left[2, G^{\prime \prime}\right]$; (c) using $M A\left[3, G^{\prime \prime}\right]$; (e) using $M A\left[4, G^{\prime \prime}\right]$. Right column shows the original noisy data and their reconstructions from (4.3). (b) $L=2$; (d) $L=3$; (f) $L=4$. 


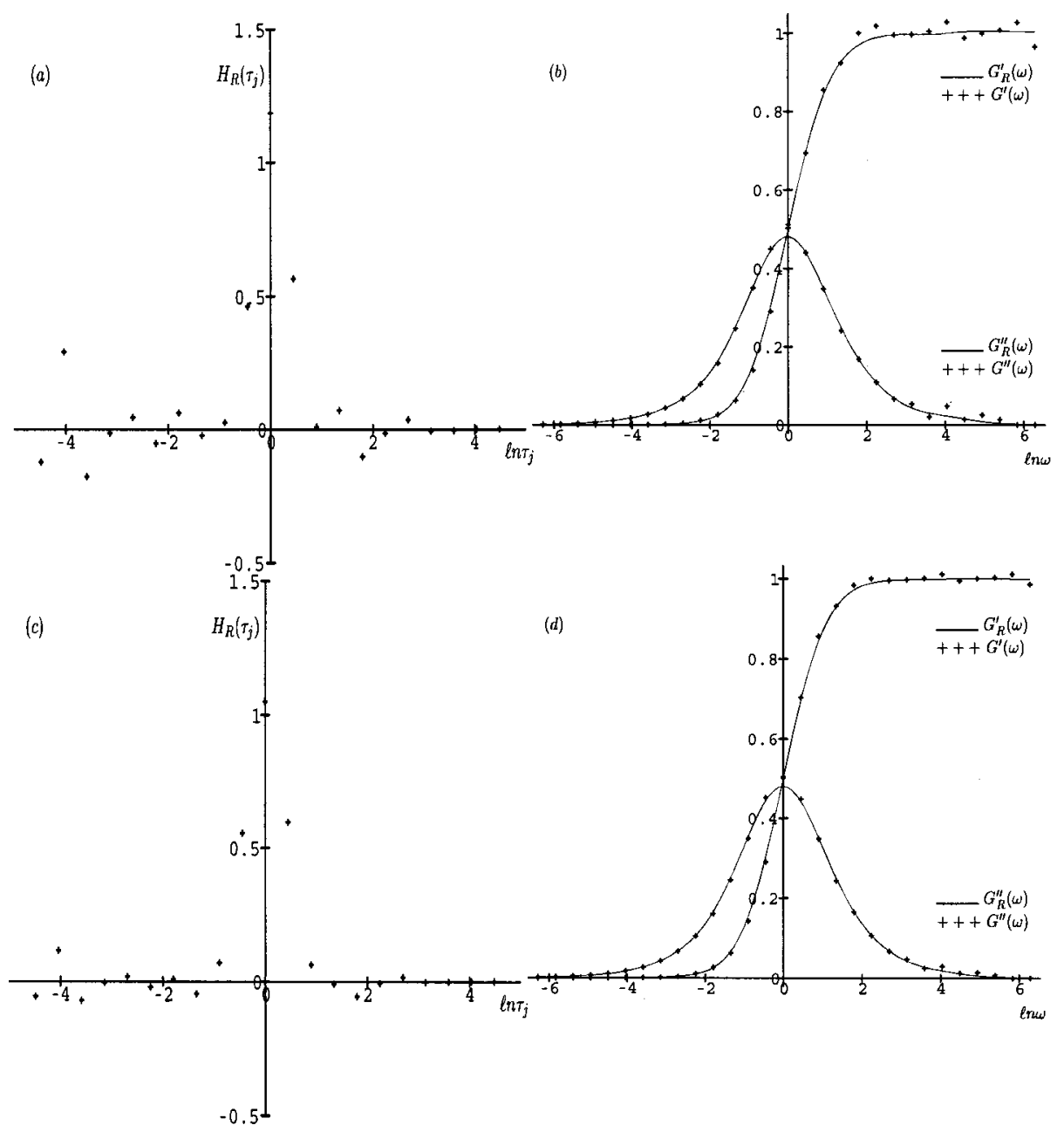

FIG. 5. Left column shows recovery of the delta function from a noisy loss modulus using $M A\left[4, G^{\prime \prime}\right]$. Right column shows the original noisy data and their reconstructions from (4.3). (a) and (b) $0.5 \%$ noise in phase lag, $1.5 \%$ noise in amplitude ratio. (c) and (d) $0.2 \%$ noise in phase lag, $0.6 \%$ noise in amplitude ratio.

$M A\left[L, G^{\prime \prime}\right]$. It is now demonstrated that, implemented correctly, both these formulae may be used to advantage to recover arbitrary spectra to within finite resolution.

The following algorithm is proposed.

(1) Exponentially sample the loss modulus $G^{\prime \prime}(\omega)$ for a sampling interval $h=\pi / 3, \pi / 5$, or $\pi / 7$. If the sampled values correspond to some other grid, use linear interpolation to produce data on the nearest $h$ grid.

(2) For the corresponding value of $L$ (namely, 2, 3, or 4) use $M A\left[L, G^{\prime \prime}\right]$ formula to generate estimates of the elastic moduli $g_{j}^{\prime \prime}$.

(3) From (68), reconstruct the storage modulus $G_{R}^{\prime}(\omega)$ on the $h$ grid.

(4) Use $M A\left[L, G_{R}^{\prime}\right]$ to generate estimates of the elastic moduli $g_{j}^{\prime}$.

(5) From (67), reconstruct the loss modulus $G_{R}^{\prime \prime}(\omega)$ on the $h$ grid.

(6) Repeat steps $2-5$ as many times as necessary, using the most recently reconstructed data at each step. 
Unless the original $G^{\prime \prime}(\omega)$ data are very noisy, only a few cycles are needed before the elastic moduli converge.

As an example of the use of this algorithm, consider the unacceptable spectrum shown in Fig. 4(e), obtained from the application of the moving-average formula $M A\left[L, G^{\prime \prime}\right]$ to the noisy data for the delta function. Using step 3 of the algorithm, the reconstructed storage modulus shown in Fig. 4(f) was obtained. It is much smoother than the original $G^{\prime}(\omega)$ data. From step 4, the spectrum shown in Fig. 6(a) was recovered. The noise in this recovered spectrum is now at an acceptable level, and there is no need for further iterations. The corresponding data reconstructions are shown in Fig. 6(b).

\section{G. Recovering the double-Gaussian spectrum of Honerkamp and Weese}

As a second application of the duality algorithm in Sec. IV F, it is applied to recover the double-Gaussian spectrum of Honerkamp and Weese (1989). The noisy oscillatory shear data published by Honerkamp and Weese are exponentially sampled on a grid with $h=\pi / 6.6$. In step 1 of the duality algorithm, it is therefore necessary to linearly interpolate the loss modulus onto an $h$ grid with $h=\pi / 7$; i.e., $L=4$. One should also note that the noise perturbations in the Honerkamp-Weese data have standard deviations proportional to the magnitude of the storage and loss moduli (4\%). Consequently, at high frequencies, the absolute noise level in the loss moduli is far less than the absolute noise level in the storage moduli. Even so, one application of $M A\left[4, G^{\prime \prime}\right]$ in step 2 of the algorithm leads to the recovery shown in Fig. 7(a). The original data and its reconstruction in step 3 are shown in Fig. 7(b). After only two cycles of the algorithm, an excellent recovery of the original spectrum is obtained, as shown in Figs. 7(c) and 7(d).

\section{DISCUSSION}

The purpose of this paper is twofold: first, to derive in an elementary manner simple moving average formulae for determining the relaxation spectrum from oscillatory shear data, and second, to demonstrate how these formulae behave when the data are contaminated with realistic levels of noise. It has been shown using synthetic noisy data that good estimates of the spectrum can be recovered if the formulae are used iteratively. Several issues remain to be addressed, and we discuss four of them here, briefly.

Non-negativity of the spectrum. A well-known theorem of Bernstein tells us that a function is completely monotonic if and only if it is the Laplace transform of a positive measure. It may be inferred that the memory kernel of a viscoelastic fluid is completely monotonic if and only if the relaxation spectrum is non-negative. The recovered estimates of the delta-function which appear in Figs. 1(a) and 2(a), however, show negative lobes, and would give rise to nonmonotonic memory kernels. (The oscillations are discernible only at very long times and on very small scales.)

The negative lobes in Figs. 1(a) and 2(a) are Gibbs-type phenomena resulting from the discontinuous nature of the delta function. As the sampling interval gets smaller the size (and number) of the negative lobes increases. The moving-average formulae, therefore, can never recover a perfect delta function from exact data, even in the limit of small sampling interval, $h$. Fortunately, if the delta function is replaced by a smooth (albeit peaked) function such as a gaussian, then for sufficiently small $h$ (of the order of the half width of the peak) the negative lobes disappear. Moreover the function may be recovered perfectly from exact data in the limit of small $h$.

Of course, it is easy to construct moving-average coefficients which preserve the non-negativity of the spectrum, either by choosing broader basis functions than deltafunctions for the spectrum, or by imposing non-negativity of the spectrum as a constraint 

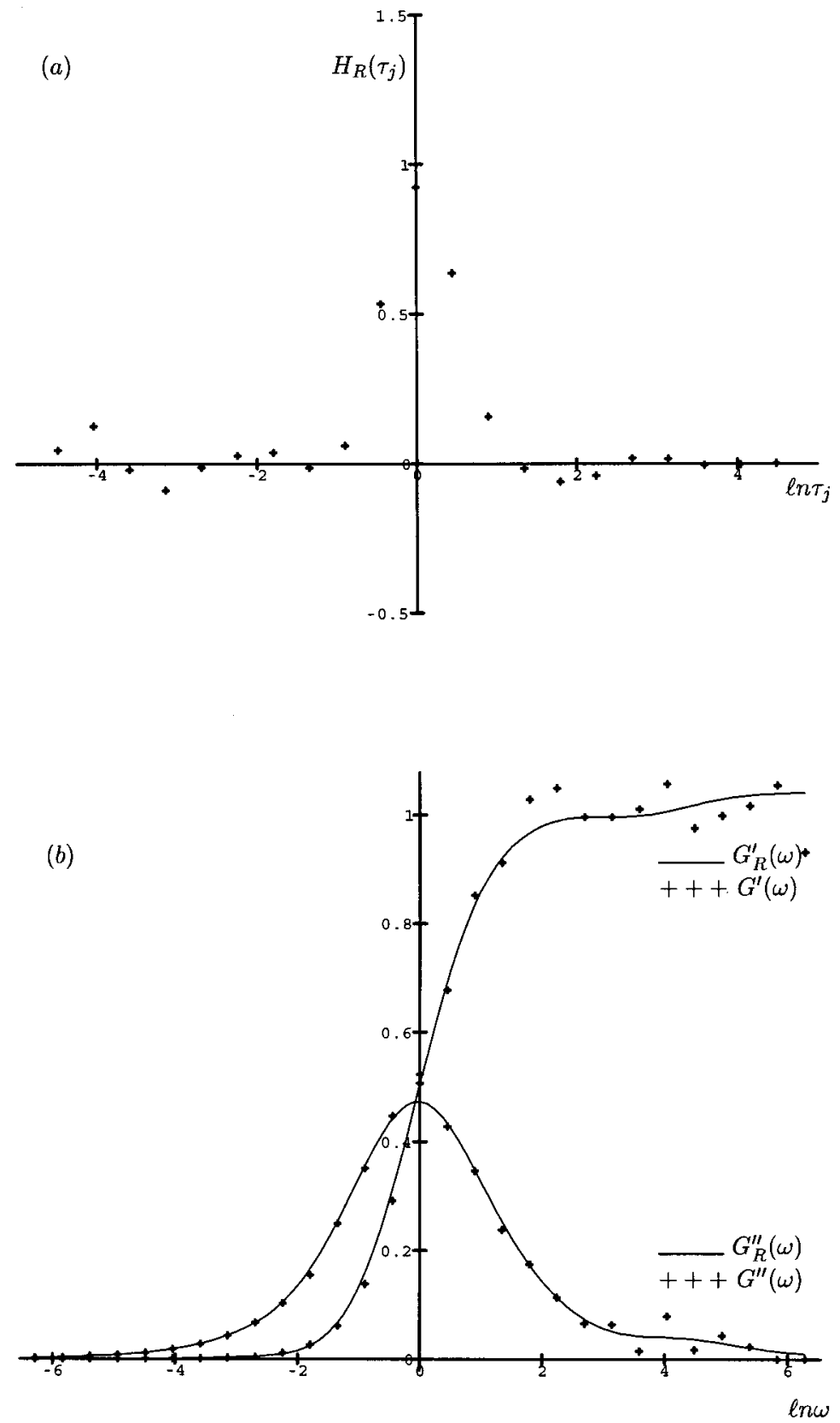

FIG. 6. (a) Recovery of the delta function from noisy loss modulus ( $1 \%$ noise in phase lag, $3 \%$ noise in amplitude ratio), after two cycles of the duality algorithm $(L=4)$. (b) Shows the original noisy data and their reconstructions after two cycles of the duality algorithm $(L=4)$.

on the least-squares solution of (60) and (61). Either way this results in a reduction in the resolving power of the formula and is therefore not optimal.

In practice, the appearance of negative elastic moduli will be the result of noise rather than a delta function in the spectrum. We have found that negative moduli can be 

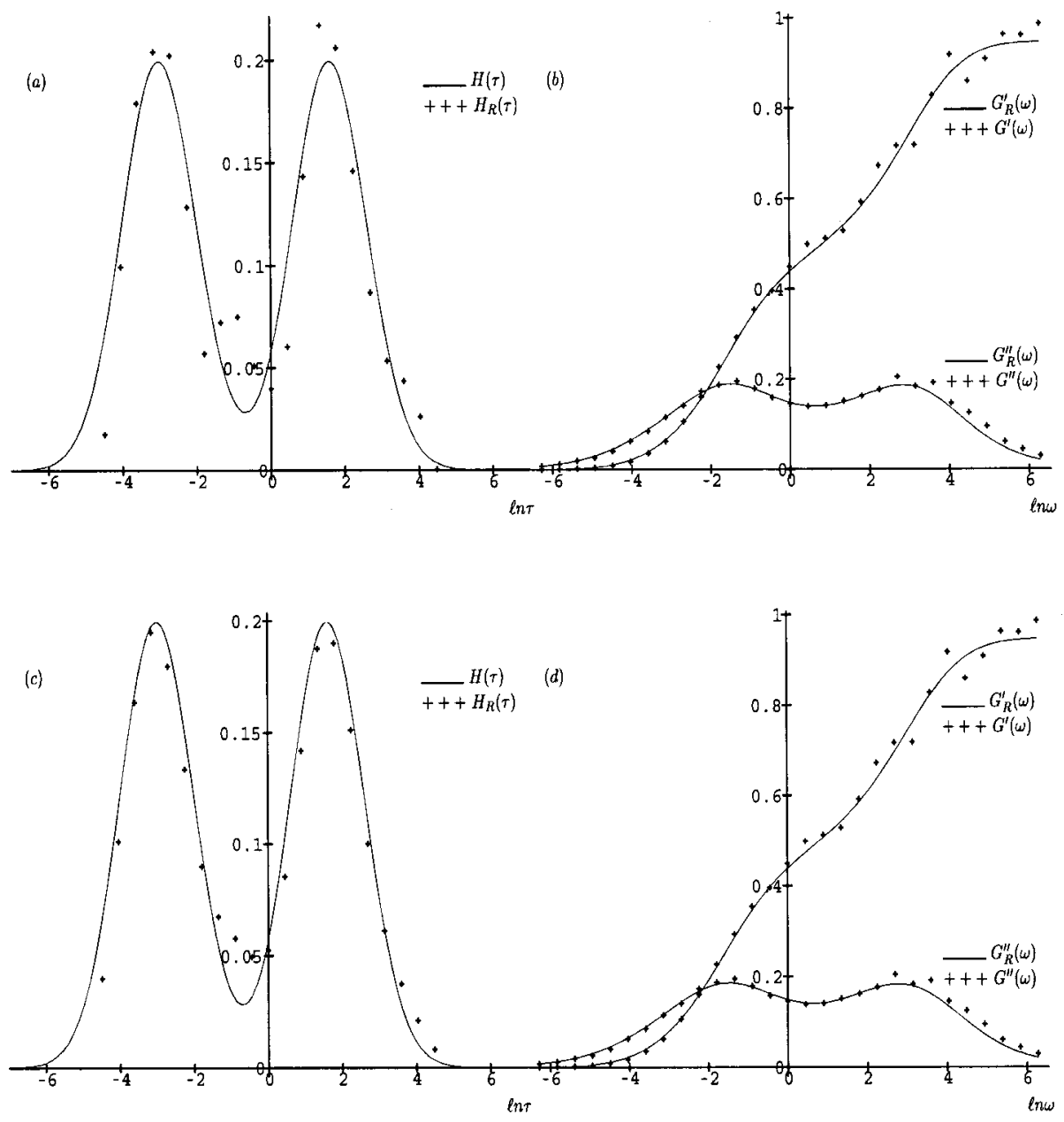

FIG. 7. Left column shows recovery of the double-Gaussian spectrum of Honerkamp-Weese from noisy loss modulus using the duality algorithm $(L=4)$. Right column shows the original noisy data and their reconstructions. (a) and (b) after 1 cycle. (c) and (d) after 2 cycles.

removed through iteration (at the cost of loss in resolution) or simply by combining neighboring positive and negative moduli, thereby preserving the sum (34).

Working with storage and loss moduli. The duality algorithm proposed in Sec. IV F works from the loss modulus. Similar duality algorithms may be devised to work from the storage modulus, or from both dynamic moduli. The performance of such algorithms on actual (in contrast to synthetic) experimental data is discussed in Davies and Anderssen (1998). Two issues are investigated in some detail, namely, the balance between resolution and noise amplification, and the effect of truncated data, i.e., sampling over too limited a frequency range.

Arbitrary sampling intervals. The moving-average formulae derived in this paper work on exponentially sampled data with a sampling interval, $h$, which is an odd integer divisor of $\pi$. Linear interpolation is recommended if the data are not available in this form. Similar moving-average formulae may be derived when $h$ is an even integer divisor of $\pi$. Newbury (1999) shows that it is possible to obtain moving average formulae for 
arbitrary values of $h$, but that, because of the phenomenon of sampling localization, the most effective use of the data is made when $h$ is an integer divisor of $\pi$ (even or odd).

Optimal number of iterations. The number of cycles used in a duality algorithm controls the balance between resolution of the spectrum and amplification of the noise in the data. Too few cycles result in insufficient filtering of the amplified noise, while too many cycles result in oversmoothing and loss of resolution in the recovered spectrum. The number of cycles, therefore, may be interpreted as another regularization parameter in the recovery of the spectrum. The question of how to choose the number of cycles optimally, so that the choice may be incorporated automatically by the algorithm, has not yet been answered. A pragmatic approach is to stop iterating once the peaks in the recovered spectrum are separated to at least the mean resolvable separation of the formula being used (see Tables III and IV).

\section{ACKNOWLEDGMENTS}

Most of this work was completed during visits between Canberra and Aberystwyth by both authors. Financial support from CSIRO and the University of Wales, Aberystwyth, is gratefully acknowledged.

\section{References}

Anderssen, R. S., F. R. de Hoog, and M. Hegland, "A stable finite difference ansatz for higher order differentiation of non-exact data," Bulletin Aust. Math. Soc. 58, 223-232 (1998).

Baumgaertel, M. and H. H. Winter, "Determination of the discrete relaxation and retardation time spectrum from dynamic mechanical data," Rheol. Acta 28, 511-519 (1989).

Brabec, C. J. and A. Schausberger, "An improved algorithm for calculating relaxation-time spectra from material functions of polymers with mondisperse and bimodal molar-mass distributions," Rheol. Acta 34, 397-405 (1995).

Carrot, C. and V. Verney, "Determination of a discrete relaxation spectrum from dynamic experimental data using the Padé-Laplace method," Eur. Polym. J. 32, 69-77 (1996).

Davies, A. R. "Optimality in regularization," in Inverse Problems in Scattering and Imaging, edited by M. Bertero and E. R. Pike (Adam Hilger, Bristol, 1992), pp. 393-410.

Davies, A. R. and R. S. Anderssen, "Sampling localization in determining the relaxation spectrum," J. NonNewtonian Fluid Mech. 73, 163-179 (1997).

Davies, A. R. and R. S. Anderssen, "Sampling localization and duality algorithms in practice," J. NonNewtonian Fluid Mech. 79, 235-253 (1998).

Emri, I. and N. W. Tschoegl, "Generating line spectra from experimental responses. Part 2: Storage and loss functions," Rheol. Acta 32, 311-321 (1993).

Engl, H. W., M. Hanke, and A. Neubauer, Regularization of Inverse Problems (Kluwer Academic, Dordrecht, 1996).

Groetsch, C. W., The Theory of Tikhonov Regularization for Fredholm Equations of the First Kind (Pitman, London, 1984).

O'Haver, T. C., An Introduction to Signal Processing in Chemical Analysis (University of Maryland Press, Baltimore, 1997).

Honerkamp, J. and J. Weese, "Determination of the relaxation spectrum by a regularization method," Macromolecules 22, 4372-4377 (1989).

Honerkamp, J. and J. Weese, "Tikhonov's regularization method for ill-posed problems," Continuum Mech. Thermodyn. 2, 17-30 (1990).

Honerkamp, J. and J. Weese, "A nonlinear regularization method for the calculation of relaxation spectra," Rheol. Acta 32, 65-73 (1993).

Mead, D. W., "Numerical interconversion of linear viscoelastic material functions," J. Rheol. 38, 1769-1795 (1994).

Morozov, V. A., "'On the solution of functional equations by the method of regularization," Sov. Math. Dokl. 7, 414-417 (1966) (English translation). 
Newbury, C. M., "Ill-posed problems in the characterization of advanced materials," Ph.D. Thesis, University of Wales, Aberystwyth, 1999.

Walters, K., Rheometry (Chapman and Hall, London, 1987).

Yanovsky, Y. G., Y. A. Basistov, and D. A. Siginer, "Linear inverse problems in viscoelastic continua and a minimax method for Fredholm equations of the first kind," Int. J. Eng. Sci. 34, 1221-1245 (1996). 